\title{
SNMMI Procedure Standard/EANM Practice Guideline for Amyloid PET Imaging of the Brain 1.0
}

\author{
Satoshi Minoshima ${ }^{1}$, Alexander E. Drzezga ${ }^{2}$ (Cochairs), Henryk Barthel ${ }^{3}$, Nicolaas Bohnen ${ }^{4}$, Mehdi Djekidel ${ }^{5}$, \\ David H. Lewis ${ }^{6}$, Chester A. Mathis ${ }^{7}$, Jonathan McConathy ${ }^{8}$, Agneta Nordberg ${ }^{9}$, Osama Sabri ${ }^{10}$, John P. Seibyl ${ }^{11}$, \\ Margaret K. Stokes ${ }^{12}$, and Koen Van Laere ${ }^{13}$ \\ ${ }^{1}$ University of Utah, Salt Lake City, Utah; ${ }^{2}$ University of Cologne, Cologne, Germany; ${ }^{3}$ University Hospital Leipzig, Leipzig, \\ Germany; ${ }^{4}$ VA Medical Center, University of Michigan, Ann Arbor, Michigan; ${ }^{5}$ Yale University, New Haven, Connecticut; \\ ${ }^{6}$ Harborview Medical Center, Seattle, Washington; ${ }^{7}$ University of Pittsburgh, Pittsburgh, Pennsylvania; ${ }^{8}$ Mallinckrodt, St. Louis, \\ Missouri; ${ }^{9}$ Karolinska Institutet, Stockholm, Sweden; ${ }^{10}$ University Hospital Leipzig, Leipzig, Germany; ${ }^{11}$ Institute for \\ Neurodegenerative Disorders, New Haven, Connecticut; ${ }^{12}$ Beth Israel Deaconess Medical Center, Boston, Massachusetts; and \\ ${ }^{13}$ University Hospital Leuven, Leuven, Belgium
}

\section{PREAMBLE}

The Society of Nuclear Medicine and Molecular Imaging (SNMMI) is an international scientific and professional organization founded in 1954 to promote the science, technology, and practical application of nuclear medicine. Its 18,000 members are physicians, technologists, and scientists specializing in the research and practice of nuclear medicine. In addition to publishing journals, newsletters, and books, the SNMMI also sponsors international meetings and workshops designed to increase the competencies of nuclear medicine practitioners and to promote new advances in the science of nuclear medicine. The European Association of Nuclear Medicine (EANM) is a professional nonprofit medical association that facilitates communication worldwide between individuals pursuing clinical and research excellence in nuclear medicine. The EANM was founded in 1985.

The SNMMI/EANM will periodically define new standards/ guidelines for nuclear medicine practice to help advance the science of nuclear medicine and to improve the quality of service to patients. Existing standards/guidelines will be reviewed for revision or renewal, as appropriate, on their fifth anniversary or sooner, if indicated. Starting February 2014, the SNMMI guidelines have been referred to as procedure standards. Any practice guideline or procedure guideline published before that date is now considered an SNMMI procedure standard.

Each standard/guideline, representing a policy statement by the SNMMI/EANM, has undergone a thorough consensus process in which it has been subjected to extensive review. The SNMMI/EANM recognizes that the safe and effective use of diagnostic nuclear medicine imaging requires specific training, skills, and techniques, as described in each document.

Received Feb. 22, 2016; accepted Feb. 22, 2016

For correspondence or reprints contact: Satoshi Minoshima, University of Utah, 30 N. 1900 E., Room 1A071, Salt Lake City, UT 84132.

E-mail: sminoshima@hsc.utah.edu

COPYRIGHT (C) 2016 by the Society of Nuclear Medicine and Molecular Imaging, Inc.

DOI: $10.2967 /$ jnumed.116.174615
The EANM and SNMMI have written and approved these standards/guidelines to promote the use of nuclear medicine procedures with high quality. These standards/guidelines are intended to assist practitioners in providing appropriate nuclear medicine care for patients. They are not inflexible rules or requirements of practice and are not intended, nor should they be used, to establish a legal standard of care. For these reasons and those set forth below, the SNMMI/EANM cautions against the use of these standards/guidelines in litigation in which the clinical decisions of a practitioner are called into question.

The ultimate judgment regarding the propriety of any specific procedure or course of action must be made by medical professionals taking into account the unique circumstances of each case. Thus, there is no implication that an approach differing from the standards/ guidelines, standing alone, is below the standard of care. To the contrary, a conscientious practitioner may responsibly adopt a course of action different from that set forth in the standards/guidelines when, in the reasonable judgment of the practitioner, such course of action is indicated by the condition of the patient, limitations of available resources, or advances in knowledge or technology subsequent to publication of the standards/guidelines.

The practice of medicine involves not only the science but also the art of dealing with the prevention, diagnosis, alleviation, and treatment of disease. The variety and complexity of human conditions make it impossible to always reach the most appropriate diagnosis or to predict with certainty a particular response to treatment. Therefore, it should be recognized that adherence to these standards/guidelines will not ensure an accurate diagnosis or a successful outcome. All that should be expected is that the practitioner will follow a reasonable course of action based on current knowledge, available resources, and the needs of the patient to deliver effective and safe medical care. The sole purpose of these standards/guidelines is to assist practitioners in achieving this objective.

\section{INTRODUCTION}

Extracellular deposition of $\mathrm{A} \beta$ peptides (or plaques) is one of the pathologic hallmarks of Alzheimer disease (AD) (1). The recent development of molecular imaging tracers that bind to $A \beta$ plaques in the brain has enabled in vivo detection of $A \beta$ plaque deposition using PET. Noninvasive detection of $A \beta$ deposition may potentially contribute to better diagnosis and management 
of patients with cognitive decline suspected of having neurodegenerative disorders. Additionally, confirmation of the presence of $\mathrm{A} \beta$ deposition among subjects and monitoring of changes in $\mathrm{A} \beta$ deposition may become critical in therapeutic interventions that are specifically designed to remove $A \beta$ deposits from the brain. As of 2015, 3 compounds had been approved for imaging $A \beta$ plaques by the U.S. Food and Drug Administration and the European Medicines Agency: ${ }^{18} \mathrm{~F}$-florbetapir (Amyvid; Eli Lilly), ${ }^{18} \mathrm{~F}$-flutemetamol (Vizamyl; GE Healthcare), and ${ }^{18}$ F-florbetaben (NeuraCeq; Piramal Pharma).

$\mathrm{AD}$ is the most common form of dementia. It is a neurodegenerative disease characterized by a constellation of clinical symptoms ranging from declines in short-term memory or executive function to behavioral changes, loss of language, alogia, impaired psychosocial function, and eventually death. The hallmarks of the disease have been classically defined by neuropathologic changes including the formation of abundant $A \beta$ plaques and neurofibrillary tangles of phosphorylated tau protein. Such protein aggregations are hypothesized to provoke or result from other pathologic processes observed in $\mathrm{AD}$, including inflammation, synaptic dysfunction, neuronal disconnection, and neuronal loss. However, the exact pathogenesis of AD and cascades of pathologic changes are still a matter of intense debate and investigation.

Recently the National Institute on Aging-Alzheimer Association and the Consortium to Establish a Registry for Alzheimer Disease, as well as the International Working Group, published updated consensus guidelines for the neuropathologic assessment of AD (2-4). These guidelines define AD as a clinicopathologic entity, instead of neuropathologic disease confirmed at autopsy, with a set of clinical signs and symptoms of cognitive and behavioral changes that are typical of patients who have substantial AD neuropathologic changes. The National Institute on Aging-Alzheimer Association consensus guidelines describe $\mathrm{AD}$ as a continuum of pathologic processes ranging from preclinical AD and mild cognitive impairment (MCI) to dementia. This has set the stage for biomarkers, including imaging biomarkers to play a role in defining and diagnosing the various time points (stages) along the $\mathrm{AD}$ continuum.

Preclinical AD is defined as the stage of the disease at which a series of pathologic events, including $A \beta$ buildup, is occurring in the brain before the onset of significant and clinically detectable symptoms.

MCI is marked by clinical symptoms of memory or other cognitive problems greater than normal for age and education. These symptoms are mild enough not to interfere with independent and instrumental activities of daily living such as dressing, eating, and caring for personal hygiene. It is important to note that MCI is a heterogeneous entity, that not everyone with MCI has $\mathrm{AD}$, and that MCI patients may or may not progress to dementia. However, the risk of conversion to clinically manifest dementia is significantly increased in MCI, and MCI can thus be considered a risk factor for the development of $\mathrm{AD}$ dementia.

Other patients who may benefit from an amyloid imaging biomarker include those with probable $\mathrm{AD}$ dementia $(5,6)$ and those with possible AD dementia (7).

\section{GOALS}

The goal of this standard/guideline is to assist nuclear medicine practitioners in recommending, performing, interpreting, and reporting the results of brain PET imaging that depicts $\beta$-amyloid $(A \beta)$ deposition in the brain (referred to as amyloid PET hereafter).

\section{COMMON CLINICAL INDICATIONS}

Appropriate-use criteria for amyloid PET have been published recently by the SNMMI and Alzheimer's Association joint task force (8-10). The appropriate-use criteria emphasize that amyloid PET is currently most likely to be helpful when the patient has objectively confirmed cognitive impairment, when the cause of cognitive impairment remains uncertain after a comprehensive evaluation by a dementia expert, when the differential diagnosis includes AD dementia, and when knowledge of the presence or absence of $A \beta$ pathology is expected to increase diagnostic certainty or alter patient management.

Dementia experts are defined as physicians trained and boardcertified in neurology, psychiatry, or geriatric medicine who devote a substantial proportion $(>25 \%)$ of patient contact time to the evaluation and care of adults with acquired cognitive impairment or dementia, including probable or suspected AD (8).

The use of amyloid PET is considered appropriate when any of the following is true: the patient has persistent or progressive unexplained $\mathrm{MCI}$; the core clinical criteria for possible $\mathrm{AD}$ are satisfied but there is an unclear clinical presentation - either an atypical clinical course or an etiologically mixed presentation; or the patient has progressive dementia and the age of onset was atypically early (usually defined as $\leq 65 \mathrm{y}$ ).

The use of amyloid PET is considered inappropriate when any of the following is true: the patient meets the core clinical criteria for probable $\mathrm{AD}$ and had a typical age of onset, there is a need to determine the severity of dementia, the patient is asymptomatic and either has a family history of $\mathrm{AD}$ or has been shown to carry the $\varepsilon 4$ allele of apolipoprotein $\mathrm{E}$, the patient has a cognitive complaint that has not been confirmed on clinical examination, a test in lieu of genotyping is needed for a patient who is a suspected autosomal dominant mutation carrier, the patient is asymptomatic, or the imaging is to be performed for nonmedical reasons (e.g., legal, insurance coverage, or employment screening).

The above appropriate-use criteria have not been validated for patient outcome or for use of possible future anti-A $\beta$ therapies, and further health services research is necessary to determine effective clinical use of amyloid PET.

\section{QUALIFICATIONS AND RESPONSIBILITIES OF PERSONNEL}

\section{A. Physician}

Amyloid PET examinations should be performed by, or under the supervision of, a physician specialized in nuclear medicine and certified by accrediting boards. Physicians who interpret amyloid PET results should also complete appropriate training programs provided by the manufacturers of approved radiotracers.

\section{B. Technologist}

Amyloid PET examinations should be performed by qualified registered or certified nuclear medicine technologists. See Performance Responsibility and Guidelines for the Nuclear Medicine Technologist for further details.

\section{PROCEDURE/SPECIFICATIONS OF THE EXAMINATION}

See also the SNMMI Procedure Standard on General Imaging. As of the end of 2014, ${ }^{18} \mathrm{~F}$-florbetapir, ${ }^{18} \mathrm{~F}$-flutemetamol, and ${ }^{18} \mathrm{~F}$-florbetaben had been approved by the U.S. Food and Drug 
Administration and the European Medicines Agency for amyloid PET examinations. Although these radiotracers share a common imaging target and similar imaging characteristics, amyloid tracers can differ in their tracer kinetics, specific binding ratios, and optimal imaging parameters (11).

\section{A. Nuclear medicine study request}

The nuclear medicine imaging facility should check with its local nuclear pharmacy provider as to the availability of the radiotracer before scheduling the examination. Advanced notice may be required for tracer delivery.

The study requisition should include clinical information about the patient to justify the study and to allow coding of the examination or study, information about the ability of the patient to cooperate with the test, and information about current medications in case mild sedation is necessary. It is also helpful to know if the patient needs to be accompanied by a guardian.

\section{B. Patient preparation and precautions}

\section{Prearrival and patient instructions}

Patients may require a careful explanation of the procedure and constant reminders of the need for their cooperation. It is often helpful to have a family member or guardian present to help with reassurance and to explain the procedure in a manner that is understood by the patient.

Patients who are unable to cooperate with the examination may need sedation. The sedation method will vary by patient and may need to be determined on the basis of the information provided by the referring physician. Sedation should be arranged at the time an amyloid PET examination is scheduled so that the procedure will go smoothly and without delay.

It is not known whether amyloid PET radiotracers have harmful fetal effects. Although pregnancy is often not relevant in a dementia population, amyloid PET should be performed on a pregnant woman only if there is a clear clinical benefit. Per standard practice, pregnancy status should be confirmed before administering a radiotracer to a female patient of reproductive potential.

Similarly, breastfeeding is rarely a concern for dementia patients. It is not known if amyloid PET tracers have harmful effects on infants or breast tissue. However, for caution in this rare instance and because of the potential for radiotracer excretion in human milk and potential radiation exposure to infants, either avoid performing amyloid PET imaging on a breastfeeding mother or have the mother interrupt breastfeeding for $24 \mathrm{~h}$ after administration of the ${ }^{18} \mathrm{~F}$ radiotracer.

There is no known evidence to suggest that there are drug interactions between amyloid radiotracers and common drugs prescribed for dementia patients, such as donepezil, galantamine, and memantine. No drug withdrawal is recommended at this time.

2. Information pertinent to the procedure

Several parameters should be taken into consideration in order to improve the quality of the study acquisition and reporting.

Correlation (preferably using digital image coregistration) with recent or concurrent morphologic imaging studies (e.g., CT or MRI) is recommended to evaluate the amount and location of brain atrophy as well as other anatomic changes such as encephalomalacia from prior stroke, brain surgery, or head trauma, which may affect amyloid PET scan interpretation.

Correlation of amyloid PET results with prior PET or SPECT brain studies may be performed, although interpretation of the amyloid PET scan should be done independently of clinical or other imaging data (other than the morphologic imaging just mentioned).
The patient's ability to lie still for the duration of the acquisition should be assessed before injection of the radiotracer.

For patients requiring sedation, ${ }^{18}$ F-labeled radiopharmaceuticals should be injected before the administration of sedation to minimize any theoretic effects of sedatives on cerebral blood flow and radiotracer delivery.

\section{Precautions}

General precautions are recommended on handling the ${ }^{18} \mathrm{~F}$ labeled radiopharmaceutical solution, such as the precaution of using aseptic technique during the injection and appropriate radiation shielding. The dose must be assayed in a suitable calibrator before administration. The injection site should be routinely inspected for dose infiltration.

Specific precautions should be taken for an amyloid PET examination. The radiopharmaceutical dose solution should be inspected before administration. It should not be used if it contains particulate matter or is discolored. The radiotracer should be injected using a short intravenous catheter $(4 \mathrm{~cm}$ [1.5 inches] or less) to minimize the potential for adsorption of substantial amounts of the drug to the catheter. Portions of the radiotracer dose may readily adhere to longer catheters.

\section{Radiopharmaceuticals}

Several radiotracers for amyloid PET have been investigated. A wealth of information is available on the radiotracer ${ }^{11} \mathrm{C}$-Pittsburgh compound $\mathrm{B}$, followed by growing numbers of publications on the ${ }^{18} \mathrm{~F}$-labeled compounds. As of 2014, ${ }^{18} \mathrm{~F}$-florbetapir (Amyvid), ${ }^{18} \mathrm{~F}$-flutemetamol (Vizamyl), and ${ }^{18} \mathrm{~F}$-florbetaben (NeuraCeq) have been approved by both the U.S. and the European authorities. Although these radiotracers share a common imaging target and similar imaging characteristics, $A \beta$ tracers can differ in their tracer kinetics, specific binding ratios, and optimal imaging parameters (11) and hence will have different recommended injected doses, times to initiate imaging after injection, and scan durations.

\section{Image acquisition}

Before scanning, patients should empty their bladder for maximum comfort during the study. They should be supine and have a suitable head support. To reduce the potential for head movement, patients should be as comfortable as possible, with the head secured completely. Tape or other flexible head restraints may be used and are often helpful. Extreme neck extension or flexion should be avoided. The entire brain should be in the field of view, including the entire cerebellum.

The imaging protocol for ${ }^{18} \mathrm{~F}$-florbetapir, ${ }^{18} \mathrm{~F}$-flutemetamol, and ${ }^{18} \mathrm{~F}$-florbetaben are described here. The radiotracer should be injected as a single intravenous slow bolus in a total volume of $10 \mathrm{~mL}$ or less. The catheter should be flushed with at least $5-15 \mathrm{~mL}$ of $0.9 \%$ sterile sodium chloride to ensure full delivery of the dose. The recommended dose, waiting period, and acquisition duration are summarized in Table 1. Images should be acquired in 3-dimensional mode with appropriate data corrections and reconstructed using attenuation correction with typical transaxial pixel sizes of $2-$ $3 \mathrm{~mm}$ and a slice thickness of $2-4 \mathrm{~mm}$.

The patient should be advised to hydrate and void after the scanning session to diminish radiation exposure.

Acquisition of early postinjection images reflecting cerebral blood flow has been described as an aid for better image interpretation and improved accuracy for ${ }^{18} \mathrm{~F}$-florbetapir (12). Such methods and their diagnostic values are currently under investigation. 


\section{E. Interpretation}

The specific criteria for amyloid PET image interpretation may differ among available radiotracers, and interpreters should be aware of the Food and Drug Administration or European Medicines Agency recommendations specific to a given amyloid tracer. The following general principles should be applied to the interpretation of amyloid PET scans.

PET images should have at least 16-bit pixels to provide an adequate range of values, and appropriate image scaling should be used for display. Gray scale display is preferred, but a specific color scale may be used, as recommended by the manufacturer for ${ }^{18} \mathrm{~F}$-flutemetamol. For ${ }^{18} \mathrm{~F}$-florbetaben and ${ }^{18} \mathrm{~F}$-florbetapir, PET images should be displayed in the transaxial orientation using gray scale or inverse gray scale. Correlated display of coronal and sagittal planes may be used to help define the tracer uptake and to ensure that the entire brain has been reviewed.

Image size should be optimized in order to evaluate gray-white matter differentiation. For ${ }^{18} \mathrm{~F}$-florbetapir, the maximum intensity of the display scale should be set to the brightest region of overall brain uptake. For ${ }^{18} \mathrm{~F}$-florbetaben, the manufacturer recommends using the white matter maximum as the reference. For ${ }^{18} \mathrm{~F}$-flutemetamol, the manufacturer recommends setting the scale intensity in the pons region to $90 \%$.

Review of transaxial images from the bottom to the top of the brain allows for initial confirmation of normal gray-white matter differentiation in the cerebellum. The cerebellar cortex is expected to be generally free of $A \beta$ deposition even in subjects with cerebral cortical amyloid disease. Thus, the cerebellar gray matter and white matter should always be clearly delineated from each other. All cerebral cortical and subcortical regions should then be screened for gray matter radiotracer uptake. Specific attention should be paid to the lateral temporal, frontal, posterior cingulate/precuneus, and parietal cortices, but also the basal ganglia. The gray matter intensity of the cerebellar cortex is usually less than that of cerebral cortical regions on a normal scan because of closer proximity of white matter structures in the latter. If image quality is significantly degraded by head motion, rescanning or rescheduling should be considered.

Negative amyloid PET scans normally show nonspecific white matter uptake and little or no binding in the gray matter. Thus, negative scans have a clear gray-white matter contrast. The amount of normal white matter uptake varies with the radiotracer used. The uptake pattern in A $\beta$-negative subjects resembles a blueprint of white matter distribution (white matter sulcal pattern) with numerous concave arboreal ramifications not reaching into the cortical ribbon. A clear, wide, irregular gap between the cerebral hemispheres will usually be visible.
Regarding positive scans, in patients with significant amounts of $A \beta$ deposition in the brain, radiotracer uptake in gray matter blurs the distinction of the gray-white junction. Thus, a key feature for distinguishing $A \beta$-positive from -negative patients is loss of gray-white matter contrast, with radiotracer uptake extending to the edge of the cerebral cortex and forming a smooth, regular boundary. Radiotracer uptake may drop sharply at the cortical margin, and a convex outer surface of the brain may be outlined rather than the white matter sulcal pattern typical of a negative scan. Gaps between the two hemispheres may no longer be defined or, if seen, may appear as a thin, regular line. Abnormal radiotracer uptake tends to be symmetric, affecting both right and left lobar structures. Cortical regions exhibiting the most distinct radiotracer accumulation in $A \beta$-positive subjects typically include the lateral temporal and frontal lobes, as well as the posterior cingulate cortex/ precuneus and the parietal lobes, whereas the sensorimotor cortex and the visual cortex can be relatively spared. Striatal radiotracer uptake, most notable in the caudate head, is also often found and may be decisive in subjects with major cortical atrophy. In patients with hereditary forms of $\mathrm{AD}$, uptake that is particularly intense in the striatum has been described (1). The cerebellar cortex does not usually show radiotracer uptake in most amyloid-positive subjects. Thus, the cerebellum can generally be used as a reference region for visual and semiquantitative interpretation. The radiotracer package inserts provide specific guidelines on the number of affected cortical regions required for definition of a positive scan. An intermediate (or indeterminate) scan pattern may also be encountered.

Some scans may be difficult to interpret because of image noise, atrophy with a thinned cortical ribbon, or image blurring. Atrophied brain may lead to false-positive results due to overestimation of radiotracer uptake in the remaining cortex based on spillover from the white matter uptake. Atrophied brain may lead to false-negative results in cases of severe atrophy, rendering it impossible to differentiate a thin ribbon of amyloid-positive cortex from adjacent white matter. The latter cases may erroneously resemble the typical appearance of nonspecific white matter uptake in a healthy control subject. When there is uncertainty as to the location or edge of gray matter on the PET scan and a coregistered CT image is available (as when the study is done on a PET/CT scanner), the interpreter should examine the CT or fused images to clarify the tissue to which radiotracer uptake localizes. If a current MRI study is available, coregistration of PET and MRI data can also provide useful information, especially on localization of cortical (gray-white matter) radiotracer uptake. The clinical introduction of hybrid PET/MRI scanners may help to further improve visual and quantitative data analysis as well as scanning procedures and diagnostic

TABLE 1

Recommended Dose, Waiting Period, and Acquisition Duration

\begin{tabular}{cccc}
\hline Radiotracer & Dose & Wait (min) & Acquisition (min) \\
\hline${ }^{18}$ F-florbetapir & $370 \mathrm{MBq}(10 \mathrm{mCi})$ & $30-50$ & 10 \\
${ }^{18}$ F-flutemetamol & $185 \mathrm{MBq}(5 \mathrm{mCi})$ & 90 & 20 \\
${ }^{18}$ F-florbetaben & $300 \mathrm{MBq}(8 \mathrm{mCi})$ & $45-130$ & 20
\end{tabular}

Variability in recommended dose is based on differences in absorbed radiation dose (Table 2). 
TABLE 2

Estimated Mean Absorbed Radiation Doses per Unit of Administered Activity

\begin{tabular}{|c|c|c|c|}
\hline \multirow[b]{2}{*}{ Organ or tissue } & \multicolumn{3}{|c|}{ Absorbed dose ( $\mu \mathrm{Sv} / \mathrm{MBq})$} \\
\hline & ${ }^{18} \mathrm{~F}$-florbetapir & ${ }^{18} \mathrm{~F}$-flutemetamol & ${ }^{18} \mathrm{~F}$-florbetaben \\
\hline Adrenal & 14 & 15 & 13 \\
\hline Brain & 10 & 12 & 13 \\
\hline Breasts & 6 & 6 & 7 \\
\hline Gallbladder wall & 143 & 287 & 137 \\
\hline Heart wall & 13 & 12 & 14 \\
\hline Kidneys & 14 & 40 & 24 \\
\hline Liver & 64 & 64 & 39 \\
\hline Lower large intestine wall & 28 & 81 & 35 \\
\hline Lungs & 9 & 17 & 15 \\
\hline Muscle & 9 & 10 & 10 \\
\hline Osteogenic cells & 28 & 13 & 15 \\
\hline Ovaries & 18 & 33 & 16 \\
\hline Pancreas & 14 & 17 & 14 \\
\hline Red marrow & 14 & 15 & 12 \\
\hline Skin & 6 & 6 & 7 \\
\hline Small intestine & 66 & 155 & 31 \\
\hline Spleen & 9 & 16 & 10 \\
\hline Stomach wall & 12 & 15 & 12 \\
\hline Testes & 7 & 5 & 9 \\
\hline Thymus & 7 & 6 & 9 \\
\hline Thyroid & 7 & 7 & 8 \\
\hline Upper large intestine wall & 74 & 173 & 38 \\
\hline Urinary bladder wall & 27 & 62 & 70 \\
\hline Uterus & 16 & 27 & 16 \\
\hline Total body & 12 & 14 & 11 \\
\hline Effective dose ( $\mu \mathrm{Sv} / \mathrm{MBq})$ & 19 & 34 & 19 \\
\hline
\end{tabular}

work-up. If a perfusion SPECT or ${ }^{18}$ F-FDG PET scan is available, correlation with areas of decreased function may assist in such uncertain cases.

Amyloid PET results should be interpreted independently of clinical information, but the final report may integrate scan findings and clinical information and suggest a final or differential diagnosis and patient management plan. Commenting on any correlation with other available imaging data may be helpful to the referring physician.

Visual interpretation comprises a qualitative binary interpretation algorithm of a positive or negative scan. Semiquantitative techniques may be helpful, including use of parametric SUV ratio images. These and other methods and their diagnostic values are currently under investigation. Absolute quantitative measurements of amyloid tracer binding in the brain using a dynamic PET imaging protocol and tracer kinetic analysis are not required clinically but may be used for research purposes.

For radiotracers that have not been approved by the Food and Drug Administration or the European Medicines Agency (e.g., ${ }^{11} \mathrm{C}$-Pittsburgh compound B), and in certain countries, imaging principles similar to those in this standard/guideline may apply.

\section{DOCUMENTATION/REPORTING}

General recommendations on nuclear medicine reports are provided in the SNMMI Procedure Standard on General Nuclear Imaging and the ACR Practice Guideline for Communication: Diagnostic Radiology.

\section{A. Indications}

The specific clinical symptoms of MCI or dementia should be documented, and the reasons for the test (e.g., uncertain clinical diagnoses, atypical age of onset, known comorbidities, or for clinical trial) should be briefly described. A management plan based on the test findings may be briefly described.

\section{B. Technique}

The name of the radiotracer used and the dose of radioactivity administered should be clearly documented, as well as the time between injection of radiotracer and scanning. Any difficulty with radiotracer injection (particularly infiltration) should also be documented. The imaging technology used (PET, PET/CT, or PET/MRI) should be noted, along with the methods of data acquisition, reconstruction, and attenuation correction. 


\section{Findings}

The pattern of radiotracer uptake in the cerebellum should be discussed, and the degree and location of cerebral atrophy (if present) should be described. If there is a loss of gray-white matter differentiation, the affected lobes should be noted. Any areas in which cerebral cortical uptake is more intense than white matter uptake should be noted.

\section{Impression}

Amyloid PET scanning depicts $A \beta$ deposition in the brain, but it is critically important to note that a scan positive for $A \beta$ deposition is not in itself indicative of AD. Positive findings can occur in non-AD forms of dementia (e.g., dementia with Lewy bodies) and other neurologic diseases and can also occur in older subjects without cognitive impairment. Also, a positive amyloid PET result does not exclude other coexistent disorders (e.g., AD plus progressive supranuclear palsy). Negative results indicate patients who are unlikely to have $\mathrm{AD}$ at the time of imaging. Negative results among MCI patients also indicate that they are unlikely to advance to AD dementia. However, negative results do not exclude the presence of a non-AD dementing illness.

The impression should state clearly whether the scan demonstrates moderate or frequent $A \beta$ deposition in the cerebral cortex (positive result) or no evidence of significant $A \beta$ deposition (negative result). An alternative would be stating that the scan demonstrates or does not demonstrate significant $A \beta$ deposition. Also, an acceptable statement may be that the findings are consistent with the presence or absence of significant $A \beta$ deposition. If the scan is indeterminate and inconclusive, this needs to be stated along with possible reasons, such as low counting rate, head motion during imaging, unexpected focal lesion, cortical atrophy, or other difficulties. The impression should not include statements such as "the scan is diagnostic of Alzheimer disease."

\section{EQUIPMENT SPECIFICATION}

Amyloid PET scans may be acquired on PET, PET/CT, or PET/ MR systems from various manufacturers. The newest-generation scanners typically offer the best image resolution and differentiation of gray matter from white matter. Adequate knowledge of the technique and equipment used is required for good quality and to avoid artifacts. For $A \beta$ brain scans, a dedicated head holder is important for positioning the head and limiting its motion. If a PET/CT or PET/MR system is not used, attenuation correction using an attenuation source or calculated attenuation correction must be used. All PET scanners need to undergo periodic quality control tests per manufacturer specifications and pass certification requirements. The SNMMI Procedure Standard on General Imaging provides more specific recommendations.

\section{QUALITY CONTROL AND IMPROVEMENT, SAFETY, INFECTION CONTROL, AND PATIENT EDUCATION CONCERNS}

The SNMMI Procedure Standard on General Imaging provides general recommendations.

Standard quality controls for every system have to be maintained per manufacturer specifications. Locally developed policies and procedures related to quality, patient education, infection control, and safety should be followed. Visual inspection is mandatory to ensure the quality of the radiotracer, which should not have any precipitation or haziness. Dose calibrator quality control should be performed at regular intervals. A dose assay of the syringe should always be performed before and after injection. The injection site should be imaged to ensure absence of dose infiltration either systematically or when a study appears noisy or shows an unexpectedly poor counting rate. This imaging option can be determined by individual sites.

Quality control standards need to be assessed to avoid misinterpretations resulting from poor image quality due to a low-count study (from poor labeling or dose infiltration), brain atrophy, bone marrow uptake in the skull, head motion, or poor head positioning.

Patient safety can be of particular concern for patients with dementia, who are often unsteady or osteoporotic or can injure themselves because of cognitive difficulties. Patients should be assisted carefully when walking in the imaging department and when getting on and off the imaging equipment. Obstacles in the room should be minimized. Sharp, unstable, or otherwise dangerous objects should be kept out of the reach of patients. When patients are being restrained on the bed or in a head holder, they should be provided with frequent explanations and reassurance to help prevent fear or resistance. Imaging of the head is optimal when the cerebrum and cerebellum are fully in the center of the axial field of view.

Sedation may be given after radiotracer injection if needed to minimize head motion, but-if the patient has cognitive impairment or dementia - only after careful medical evaluation. Sedated patients will need closer monitoring during and after the study until the effects have worn off. Sometimes paradoxic activation occurs in elderly patients. However, sedation should be avoided in elderly patients as much as possible.

Patients may have difficulty understanding the reason for the procedure. A family member or guardian can sometimes assist communication and compliance with instructions during the examination.

The risk of adverse events caused by the radiotracer is typically low, at less than $2 \%$, and may include headaches, nausea, dizziness, flushing, increased blood pressure, musculoskeletal pain, or an injection site reaction. The individual package inserts provide full side effect profiles.

\section{RADIATION SAFETY IN IMAGING}

It is the position of SNMMI that exposure of patients to ionizing radiation should be at the minimum level consistent with obtaining a diagnostic examination (see the SNMMI Procedure Standard on General Imaging). Currently approved amyloid PET tracers offer a reasonable compromise between radiation exposure following ALARA (as low as reasonably achievable) principles and image quality. The radiation exposure from an amyloid PET study - at about 4-7 mSv-is within the range of commonly performed imaging studies. Radiation dosimetry estimates in humans have been evaluated in pilot studies for ${ }^{18} \mathrm{~F}$-florbetapir and ${ }^{18} \mathrm{~F}$-flutemetamol $(13,14)$, as well as in the ${ }^{18} \mathrm{~F}$-florbetapir (15) and ${ }^{18} \mathrm{~F}$-flutemetamol clinical trials. Florbetaben dosimetry has been previously published (16).

\section{ACKNOWLEDGMENTS}

The Committee on SNMMI Procedure Standards consists of the following individuals: Kevin J. Donohoe, MD (Chair) (Beth Israel Deaconess Medical Center, Boston, MA); Bennett S. Greenspan, MD, MS (Medical College of Georgia/Augusta 
University, Augusta, GA); Helena Balon, MD (Beaumont Health System, Royal Oak, MI); Twyla B. Bartel, DO, MBA (Global Advanced Imaging, PLLC); David Brandon, MD (Emory University/Atlanta VA, Atlanta, GA); Paul E. Christian, CNMT, BS, PET (Huntsman Cancer Institute, University of Utah, Salt Lake City, UT); Dominique Delbeke, MD (Vanderbilt University Medical Center, Nashville, TN); Vasken Dilsizian, MD (University of Maryland Medical Center, Baltimore, MD); James R. Galt, PhD (Emory University Hospital, Atlanta, GA); Jay A. Harolds, MD (OUHSC-Department of Radiological Science, Edmond, OK); Aaron Jessop, MD (UT M.D. Anderson Cancer Center, Houston, TX); David H. Lewis, MD (Harborview Medical Center, Seattle, WA); Alan B. Packard, PhD (Boston Children's Hospital, Boston, MA); J. Anthony Parker, MD, PhD (Beth Israel Deaconess Medical Center, Boston, MA); James A. Ponto, RPh, BCNP (University of Iowa, Iowa City, IA); Lynne T. Roy, CNMT (Cedars/Sinai Medical Center, Los Angeles, CA); Heiko Schoder, MD (Memorial Sloan Kettering Cancer Center, New York, NY); Barry L. Shulkin, MD, MBA (St. Jude Children's Research Hospital, Memphis, TN); Michael G. Stabin, PhD (Vanderbilt University, Nashville, TN); and Mark Tulchinsky, MD (Milton S. Hershey Med Center, Hershey, PA).

The EANM Neuroimaging Committee consists of the following individuals: M. Pagani (Chair) (Institute of Cognitive Sciences and Technologies, Italy); S. Hesse (Vice Chair) (University of Leipzig, Germany); G. Chételat (Centre Cyceron, France); E. Van De Giessen (University of Amsterdam, The Netherlands); V. Garibotto (Geneva University and Geneva University Hospitals, Switzerland); S. Pappata (Institute of Biostructure and Bioimaging [IBB], Italy); A. Drzezga (University Hospital of Cologne, Germany); I. Law, (Rigshospitalet, Denmark); A.A. Lammertsma (VU University Medical Center, The Netherlands); P. Payoux (CHU Purpan, France); S. Morbelli (IRCCS San Martino-IST, University of Genoa, Italy); and J. Arbizu (University of Navarra, Spain).

\section{REFERENCES}

1. Villemagne VL, Ataka S, Mizuno T, et al. High striatal amyloid beta-peptide deposition across different autosomal Alzheimer disease mutation types. Arch Neurol. 2009;66:1537-1544.
2. Montine TJ, Phelps $\mathrm{CH}$, Beach TG, et al. National Institute on Aging-Alzheimer's Association guidelines for the neuropathologic assessment of Alzheimer's disease: a practical approach. Acta Neuropathol (Berl). 2012;123:1-11.

3. Hyman BT, Phelps $\mathrm{CH}$, Beach TG, et al. National Institute on AgingAlzheimer's Association guidelines for the neuropathologic assessment of Alzheimer's disease. Alzheimers Dement. 2012;8:1-13.

4. Dubois, Feldman HH, Jacova C, et al. Advancing research diagnostic criteria for Alzheimer's disease: the IWG-2 criteria. Lancet Neurol. 2014;13:614-629.

5. Klunk WE. Amyloid imaging as a biomarker for cerebral beta-amyloidosis and risk prediction for Alzheimer dementia. Neurobiol Aging. 2011;32(suppl 1):S20-S36.

6. Dubois B, Feldman HH, Jacova C, et al. Research criteria for the diagnosis of Alzheimer's disease: revising the NINCDS-ADRDA criteria. Lancet Neurol. 2007;6:734-746.

7. McKhann GM, Knopman DS, Chertkow H, et al. The diagnosis of dementia due to Alzheimer's disease: recommendations from the National Institute on Aging Alzheimer's Association workgroups on diagnostic guidelines for Alzheimer's disease. Alzheimers Dement. 2011;7:263-269.

8. Johnson KA, Minoshima S, Bohnen NI, et al. Update on appropriate use criteria for amyloid PET imaging: dementia experts, mild cognitive impairment, and education. J Nucl Med. 2013;54:1011-1013.

9. Johnson KA, Minoshima S, Bohnen NI, et al. Appropriate use criteria for amyloid PET: a report of the Amyloid Imaging Task Force, the Society of Nuclear Medicine and Molecular Imaging, and the Alzheimer's Association. J Nucl Med. 2013;54:476-490.

10. Johnson KA, Minoshima S, Bohnen NI, et al. Appropriate use criteria for amyloid PET: a report of the Amyloid Imaging Task Force, the Society of Nuclear Medicine and Molecular Imaging, and the Alzheimer's Association. Alzheimers Dement. 2013;9:e-1-e-16.

11. Rowe CC, Villemagne VL. Brain amyloid imaging. J Nucl Med. 2011;52:1733-1740.

12. Hsiao IT, Huang CC, Hsieh CJ, et al. Correlation of early-phase ${ }^{18} \mathrm{~F}$-florbetapir (AV-45/Amyvid) PET images to FDG images: preliminary studies. Eur J Nucl Med Mol Imaging. 2012;39:613-620.

13. Lin KJ, Hsu WC, Hsiao IT, et al. Whole-body biodistribution and brain PET imaging with $\left[{ }^{18} \mathrm{~F}\right] \mathrm{AV}-45$, a novel amyloid imaging agent: a pilot study. Nucl Med Biol. 2010;37:497-508.

14. Koole M, Lewis DM, Buckley C, et al. Whole-body biodistribution and radiation dosimetry of ${ }^{18} \mathrm{~F}-\mathrm{GE} 067$ : a radioligand for in vivo brain amyloid imaging. $\mathrm{J} \mathrm{Nucl}$ Med. 2009;50:818-822.

15. Highlights of Prescribing Information [Amyvid; page 5]. Eli Lilly and Company website. http://pi.lilly.com/us/amyvid-uspi.pdf. Accessed February 29, 2016.

16. O'Keefe GJ, Saunder TH, Ng S, et al. Radiation dosimetry of beta-amyloid tracers ${ }^{11} \mathrm{C}-\mathrm{PiB}$ and ${ }^{18} \mathrm{~F}-\mathrm{BAY} 94-9172$. J Nucl Med. 2009;50:309-315.

\section{APPROVAL}

This standard/guideline was approved by the Board of Directors of the SNMMI on January 30, 2016, and by the EANM Board on February 10, 2016. 